\title{
The effect of straight chimney temperature on pollutant dispersion
}

\author{
Fida Zair ${ }^{1, *}$, Mhamed Mouqallid ${ }^{2}$, and El houssine Chatri ${ }^{1}$ \\ ${ }^{1}$ Laboratory of Atomic, Mechanical, Photonic and Energetic, Faculty of Sciences, Moulay Ismail University, Physics Departement, \\ Meknes, Morocco \\ ${ }^{2}$ Department of Energy, ENSAM Meknes, Moulay Ismail University, Meknes, Morocco
}

\begin{abstract}
Air pollution is considered one of the most important contemporary problems that threaten a person's life and environment. Among the major sources of air pollution are pollutants emitted from smokestacks. The objective of this work is to numerically study the dispersion of a pollutant ejected from a chimney into an air stream. The mass, momentum and energy conservation equations are solved using the finite volume method (MFV) and numerical simulations are performed using Ansys Fluent CFD software. Two case studies were discussed: The first case study is to study the influence of the direction of the wind speed and in the last case study, the temperature evolution on the dispersion of the ejected plume particles. The study demonstrated the extent of the dispersion in relation to these two parameters. The results obtained confirm the need to improve filtering systems in order to reduce the risks attributed to discharges and to propose solutions to manage the effect of these discharges on the environmental population.
\end{abstract}

\section{Introduction}

Air pollution is a very complex phenomenon given the diversity of pollutants, their combination and their modification in the atmosphere. The evolution of these contaminants is difficult to understand rigorously, this complexity contributes to heightened concerns about air quality. Emissions from industrial chimneys are a major source of air pollution and their dispersion depends on several parameters in particular.

Previous work in this area has focused on determining the influence of these parameters on the dispersion phenomenon. A study on the parameters influencing the dispersion of gaseous pollutants was carried out by Diaf and Bouchaour [1] in which they stated that the dispersion of pollutants in the atmosphere is essentially carried out in the atmospheric boundary layer: the most disturbed layer, constantly agitated by turbulent movements. This dispersion is influenced by several parameters such as wind speed, atmospheric stability and thermal state.

The results found show that the dispersion of gaseous pollutants through the atmosphere is very complex. Thus, an anticyclonic situation (very light winds) favours high levels of pollution because it leads to an accumulation of gases.

The inversion of the vertical thermal gradient has the same consequences. In the opposite, a low-pressure situation (i.e. more sensitive winds) allows a better dilution of pollutants in the atmosphere.

Benkoussas and Bouhdjar [2] conducted a study on the atmospheric dispersion of effluents emitted by the chimneys of a cement plant. The study is conducted in numerical form and aims to determine the effects of the following factors (wind speed, stack height, environmental roughness) on the diffusion of particles in the ejected plume.

This paper is founded on earlier work by Smith and Frankenberg [3] which showed that stack height influences dispersion under thermal inversion (when a pollutant is emitted from a low stack opening below the thermal inversion layer, there is an accumulation of the thermal inversion layer below, but if the stack opens above it, the pollutants diffuse normally).

Wilson [4] and Vincent's [5] studies of the atmospheric boundary layer. They highlighted the influence of obstacles on the dispersion of pollutants. They found that the dispersion is more pronounced for larger injection ratios $(\mathrm{R}=$ (ejection velocity) $($ wind speed)) and they found that the height reached by the particles in the vicinity of the outlet is significant when $\mathrm{R}$ increases. When $\mathrm{R}$ is low (wind speed increases) the dispersion of particles will be similarly low while the concentration is relatively high. The high crosswind speed favours dilution of the plume.

In addition, stack elevation affects the dispersion of releases. Ejection at a higher elevation generates a larger plume that promotes dilution. The higher the height, the further away the maximum concentration moves and leaves the domain.

Menaouer [6] studied a numerical model of the evolution of the dispersion of pollutants emitted by a stack. This work is a numerical approach employed to define the fields of continuous or instantaneous

Corresponding author: f.zair@edu.umi.ac.ma 
dispersion of pollutants by industrial stacks, the results of which help in decision-making and determine the risk areas, therefore, the choice of a plant location. This research also permits to define the effect of different physical parameters on the dispersion phenomenon, for example wind velocity and the roughness of the environment.

Numerical simulations of atmospheric dispersion under the ANSYS FLUENT 12.1 allow to plot the flow characteristics (velocities, concentrations, etc.) and to detect the characteristics of the plume in a neutral situation. The results obtained are plotted and are in very good accordance with those in the literature.

Flowe and Kumar [7] conducted a parametric study to determine the length of the recirculation cavity according to the ratio of the width of the building to the height of the building, both at the front and at the back of the building. The main purpose of this investigation was to assess the viability of utilizing a three-dimensional k- $\varepsilon$ digital model to model airflow beyond the geometry of the building and chimney. The dispersive data collected was then used to determine new correlations between the ratio of building width to height, the size of the recirculation cavity and the medium concentration in the subsequent recirculation cavity of the building.

Liu and Ahmadi [8] have studied the movement, dispersion and deposition of particles in the vicinity of a building using a Lagrangian particle survey approach. The computational model used reported the forces of train and uplift acting on the particles as in addition to the influence of Brownian motion and the effects of gravitational sedimentation. A point source of helium was selected as the contaminant source, and the helium concentration in the posterior plane of the building, perpendicular to the direction of air flow, was evaluated. The results found that the diffusion and deposition of particles of $0.01 \mu \mathrm{m}$ and $1 \mu \mathrm{m}$ were comparable, and that the gravitational force had a significant impact on the deposition rate of particles of $10 \mu \mathrm{m}$. Comparison of the results with the available data revealed agreement on the average airflow and the average gas concentration.

Olvera et al [9] studied the posterior recirculation cavity of a cubic building at using a commercial CFD code and the turbulence model k- $\varepsilon$ RNG. They found that plume flotation affects the size and form of the cavity area of the flow profile and the concentrations within this area. In their study report, the authors recommended including this effect in the plume folding algorithm to improve the accuracy of far-field concentration distribution modelling results.

This would be essential for accident investigations, when accurate predictions of near-field concentration fluctuations near the source over short periods of time are required.

DiSabatino et al [10] studied the effect of the Sct on airflow in a small building configuration and on the dispersion of pollutants in urban canyons. To this end, they compared the standard k- $\varepsilon$ model with the ADMSUrban atmospheric dispersion model. Like previous researchers, they found that concentrations were overestimated in urban canyons, a phenomenon they attributed to the low values for turbulent kinetic energy found near buildings with CFD simulations. They further indicated that dispersion could be artificially increased by reducing the Sct.

Tominaga and Stathopoulos [11] investigate different models of airflow turbulence and pollutant dispersion on an isolated cubic obstacle. They further discovered that the standard $k-\varepsilon$ model did not predict concentrations because it does not replicate the basic components of flow structures, for example, opposite flow on the roof of a building. The k- $\varepsilon$ RNG model and the k- $\varepsilon$ Realizable model were in accord with the experimental data for Sct $=0.3$. This allowed the authors to confirm that the underestimation of turbulent pulse scattering in the wind flow could be compensated for by a lower Sct value.

The objective of our paper is to study the influence of some parameters on the dispersion of pollutants emitted by straight chimneys: wind speed direction and jet temperature.

\section{Methodology}

\subsection{Governing Equation}

Considering the flow from a chimney. The chimney emits a mixture of air and smoke at $U_{\text {chimney }}$ speed and $\mathrm{T}_{\text {chimney }}$ temperature. This plume is subjected to a wind speed of $U_{\text {wind, }}$ the ambient temperature is $T_{\text {wind }}$.

The governing equations are based on the continuity and the momentum equations in steady state. The fluctuating velocity components were averaged according to Reynolds Navies- Stokes decomposition RANS were $U_{i}=u_{i}+u_{i}^{\prime}$ That maybe written for the mean field as:

$$
\begin{aligned}
& \frac{\partial u_{i}}{\partial x_{i}}=0 \\
& u_{j} \frac{\partial u_{i}}{\partial x_{j}}=-\frac{1}{\rho} \frac{\partial P}{\partial x_{i}}+\frac{\partial}{\partial x_{j}}\left(v \frac{\partial u_{i}}{\partial x_{j}}-\overline{u_{i}^{\prime} u_{j}^{\prime}}\right) \\
& \text { With: }-\overline{u_{i}^{\prime} u_{j}^{\prime}}=v_{t}\left(\frac{\partial U_{i}}{\partial x_{j}}+\frac{\partial U_{j}}{\partial x_{i}}\right)-\frac{2}{3} \kappa \delta_{i j}
\end{aligned}
$$

Where $\kappa$ is the turbulent kinetic energy defined by:

$$
\kappa=\frac{1}{2} \overline{u_{\mathrm{i}}^{\prime} \mathrm{u}_{\mathrm{j}}^{\prime}}
$$

The term $\overline{\mathrm{u}_{\mathrm{i}}^{\prime} \mathrm{u}_{\mathrm{j}}^{\prime}}$ identifies the Reynolds stresses tensor. This additional term is take into account through new equations that require closure or turbulence models.

\subsection{Turbulence Modeling}

The mathematical models used are turbulence models with two equations of the first order $(\kappa-\varepsilon$ standard, $\kappa-\varepsilon$ RNG, $\kappa-\varepsilon$ Realizable, $\kappa-\omega$ and $\kappa-\omega$ SST) and the 
second order RSM. All models are tested and give the same results when found, but in short, we just presented the RNG $\kappa-\varepsilon$ model.

As introduced by Launder and Spalding [12], the RNG $\kappa-\varepsilon$ model were applied using the eddy viscosity $v_{\mathrm{t}}$ model schemes given by:

$$
v_{t}=C_{v} \frac{\kappa^{2}}{\varepsilon}
$$

Where $\varepsilon$ is the kinetic energy dissipation rate taken as:

$$
\varepsilon=v \frac{\overline{\partial^{2} U_{i}}}{\partial x_{j}^{2}}
$$

For the RNG $\kappa-\varepsilon$ model the turbulent kinetic energy $\kappa$ and the kinetic energy dissipation $\varepsilon$ are given by the following equations

- Turbulent kinetic energy transport equation $\kappa$

$$
\frac{\partial \kappa}{\partial t}+U_{i} \frac{\partial \kappa}{\partial x_{i}}=\frac{\partial}{\partial x_{i}}\left(\left(\alpha_{\kappa} v_{\text {eff }}\right) \frac{\partial \kappa}{\partial x_{i}}\right)+P_{\kappa}-\varepsilon
$$

- The kinetic energy dissipation equation $\varepsilon$

$$
\frac{\partial \varepsilon}{\partial \mathrm{t}}+\mathrm{U}_{\mathrm{i}} \frac{\partial \varepsilon}{\partial \mathrm{x}_{\mathrm{i}}}=\frac{\partial}{\partial \mathrm{x}_{\mathrm{j}}}\left(\left(\alpha_{\varepsilon} v_{\text {eff }}\right) \frac{\partial \varepsilon}{\partial \mathrm{x}_{\mathrm{i}}}\right)+\mathrm{C}_{\varepsilon 1} \mathrm{P}_{\kappa} \frac{\varepsilon}{\kappa}-\mathrm{C}_{\varepsilon 2} \frac{\varepsilon^{2}}{\kappa}-\mathrm{R}_{\varepsilon}
$$

$$
\text { With: } P_{\kappa}=v_{t}\left(\frac{\partial U_{i}}{\partial x_{j}}+\frac{\partial U_{j}}{\partial x_{i}}\right) \frac{\partial U_{i}}{\partial x_{j}}
$$

With: $\mathrm{C}_{v}, \mathrm{C}_{\varepsilon 1}$ and $\mathrm{C}_{\varepsilon 2}$ are the empirical coefficients and $\sigma_{\kappa}$ and $\sigma \varepsilon$ are the turbulent Prandtl numbers for $\kappa$ and $\varepsilon$ respectively. $\mathrm{R}_{\varepsilon}$ is the additional term related to the shear deformation velocity.

The constants of the RNG model are known.

\section{Geometry of the Calculation Domain and Boundary Conditions}

In Fig. 1 we consider a straight chimney with a height $\mathrm{h}=$ $0,10 \mathrm{~m}$ and a diameter $\mathrm{d}=0,01 \mathrm{~m}$. The jet ejected from the chimney and the transverse flow contain air, the ratio between the ejection velocity and that of the transverse flow is equal to $R=1.6$. The calculation range is wide enough so that the boundaries of the range do not disturb the flow.

The flow topology requires a very fine mesh in a large part of the domain. In order to obtain a precise description of all variations, especially those closest to the chimney. We have adopted a non-uniform mesh size. To do this, the commercial code CFD ANSYS/FLUENT was used, which was able to simulate the dispersion phenomenon using the finite volume method presented by Patankar [13].

For the discretization schemes, we have adopted the following schemes:

The "First Order Upwind" diagram for the turbulence kinetic energy equations, the dissipation equation, the motion quantity equation and the energy equation. For pressure-speed coupling, we adopted the SIMPLE algorithm, for coupling between the equations for conserving the amount of motion and the continuity equation.

To complete the problem, in addition to the systems of equations mentioned above, the boundary conditions summarized in the following table (Table 1) must be taken into account:

Table 1. Boundary conditions

\begin{tabular}{|c|c|c|c|}
\hline & Chimney & Wind & $\begin{array}{c}\text { Chimney wall, } \\
\text { ground }\end{array}$ \\
\hline Velocity & $\mathrm{U}=0$ & $\mathrm{U}=\mathrm{U}_{\text {wind }}$ & $\mathrm{U}=0$ \\
& $\mathrm{~V}=\mathrm{U}_{\text {chimney }}$ & $\mathrm{V}=0$ & $\mathrm{~V}=0$ \\
\hline Temperature & $\mathrm{T}=\mathrm{T}_{\text {chimney }}$ & $\mathrm{T}=\mathrm{T}_{\text {wind }}$ & $\frac{\partial \mathrm{T}}{\partial \mathrm{n}}=0$ \\
\hline
\end{tabular}

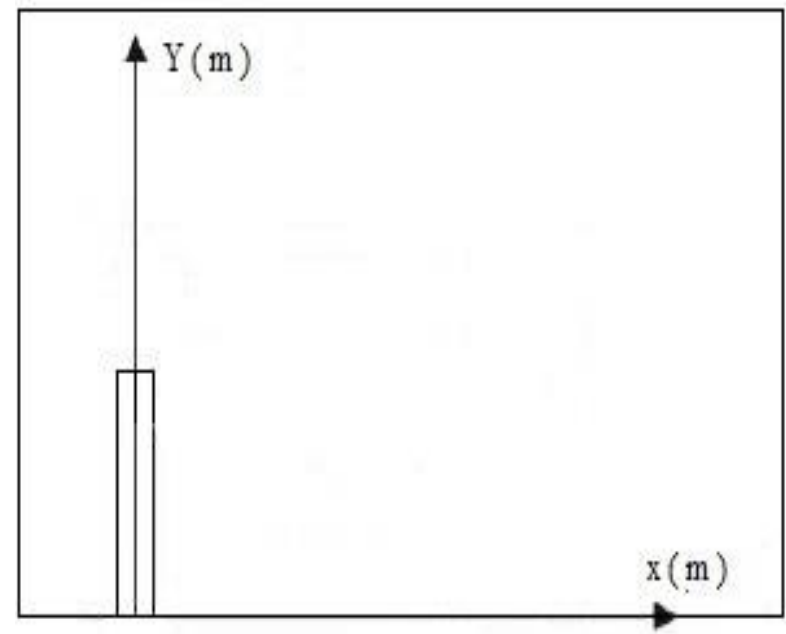

Figure 1. Geometry diagram

\section{Results and Interpretations}

\subsection{Validation of Results}

The results of the validation are plotted for longitudinal (U) velocity profiles using the same conditions of Mahjoub and Al [14]. The numerical simulation is tested for an incompressible and unsteady flow.

Figures 2-4 show the velocity profiles for different cross-sections $\mathrm{x}(\mathrm{x}=-0.005 \mathrm{~m} ; \mathrm{x}=0 \mathrm{~m}$ and $\mathrm{x}=0.008 \mathrm{~m})$ of the flow. These figures show a good agreement between the results found and those of Mahjoub and $\mathrm{Al}$ [14]. 


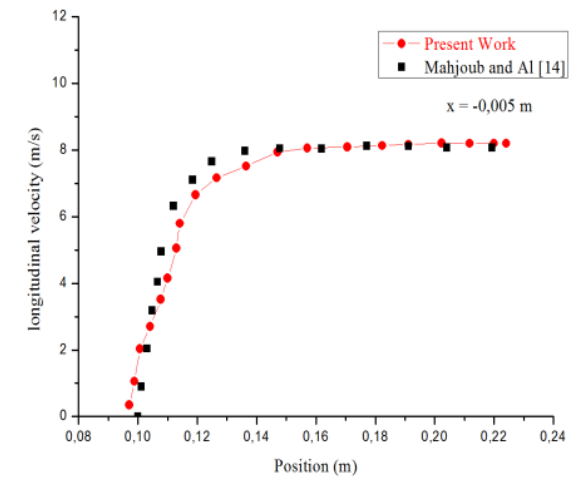

Figure 2. Longitudinal velocity profile for position $\mathrm{x}=-0.005 \mathrm{~m}$

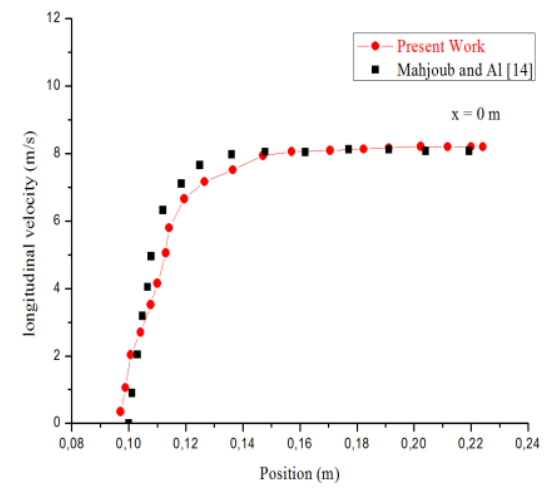

Figure 3. Longitudinal velocity profile for position $\mathrm{x}=0 \mathrm{~m}$

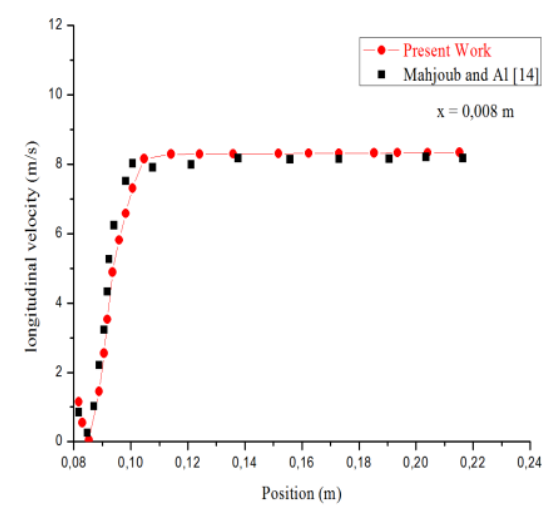

Figure 5. Longitudinal velocity profile for position $\mathrm{x}=0.008 \mathrm{~m}$

\subsection{Discussion}

\subsubsection{The Effect of Wind Velocity Direction}

To study the influence of wind speed direction on pollutant dispersion, the wind direction has been changed $\left(\alpha=0^{\circ}, \alpha=15^{\circ}, \alpha=20^{\circ}, \alpha=30^{\circ}, \alpha=45^{\circ}, \alpha=60^{\circ}\right.$ and $\alpha=75^{\circ}$ ) and for the ejection speed will be fixed at 8 $\mathrm{m} / \mathrm{s}$, while the wind speed is equal to $5 \mathrm{~m} / \mathrm{s}$. Depending on these directions, the dispersion results were obtained in an area $\mathrm{x}=0.2 \mathrm{~m}$ from the chimney (downstream of the stack). In Fig.5 and 6, we present the variation in longitudinal and transverse velocity as a function of $\mathrm{Y}$, respectively.

It should be noted that the speed curve remains the same for all seven values of $\alpha$ and that, the greater the direction angle, the higher the dispersion of pollutants.

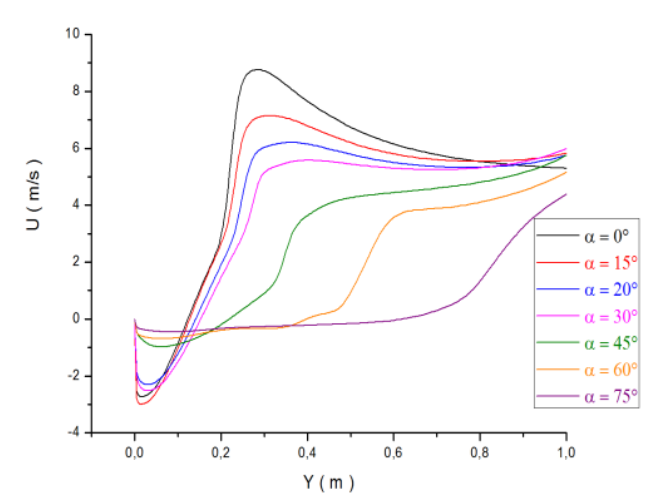

Figure 6. Evolution of the longitudinal velocity at $\mathrm{x}=0.2 \mathrm{~m}$ for a straight chimney

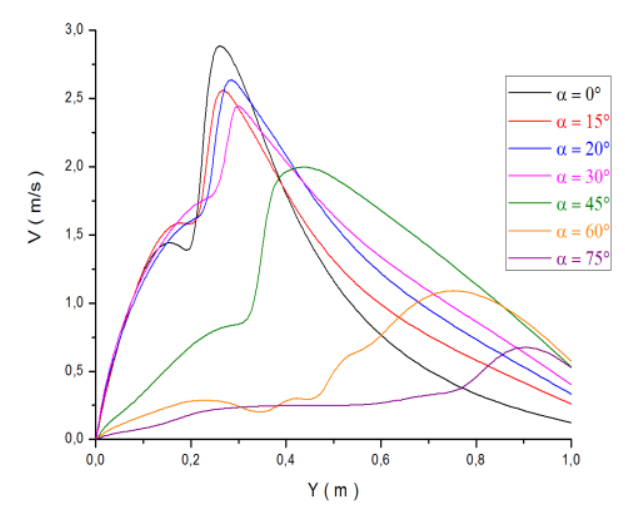

Figure 4. Evolution of the transverse velocity at $\mathrm{x}=0.2 \mathrm{~m}$ for a straight chimney

\subsubsection{The Evolution of the Jet Temperature}

This section keeps the same ratio of speeds $\mathrm{R}$ which is equal to 1.6. We trace the evolution of the temperature according to $\mathrm{Y}$. To do this, one moves away from the chimney each time by a distance $\mathrm{x}(\mathrm{x}=0.2,0.4,0.6,1.2,2$ and $2,5 \mathrm{~m}$ ) and one traces the value of the temperature on function of $Y$.

For the curve 7 , the temperature change starts from $Y$ $=0.1 \mathrm{~m}$, which means that we do not have a decent pollutant. All curves have the same speed but, when moving away from the stack, the maximum temperature of the pollutant decreases. In addition, when the ejection temperature is increased, the maximum temperature value increases. 

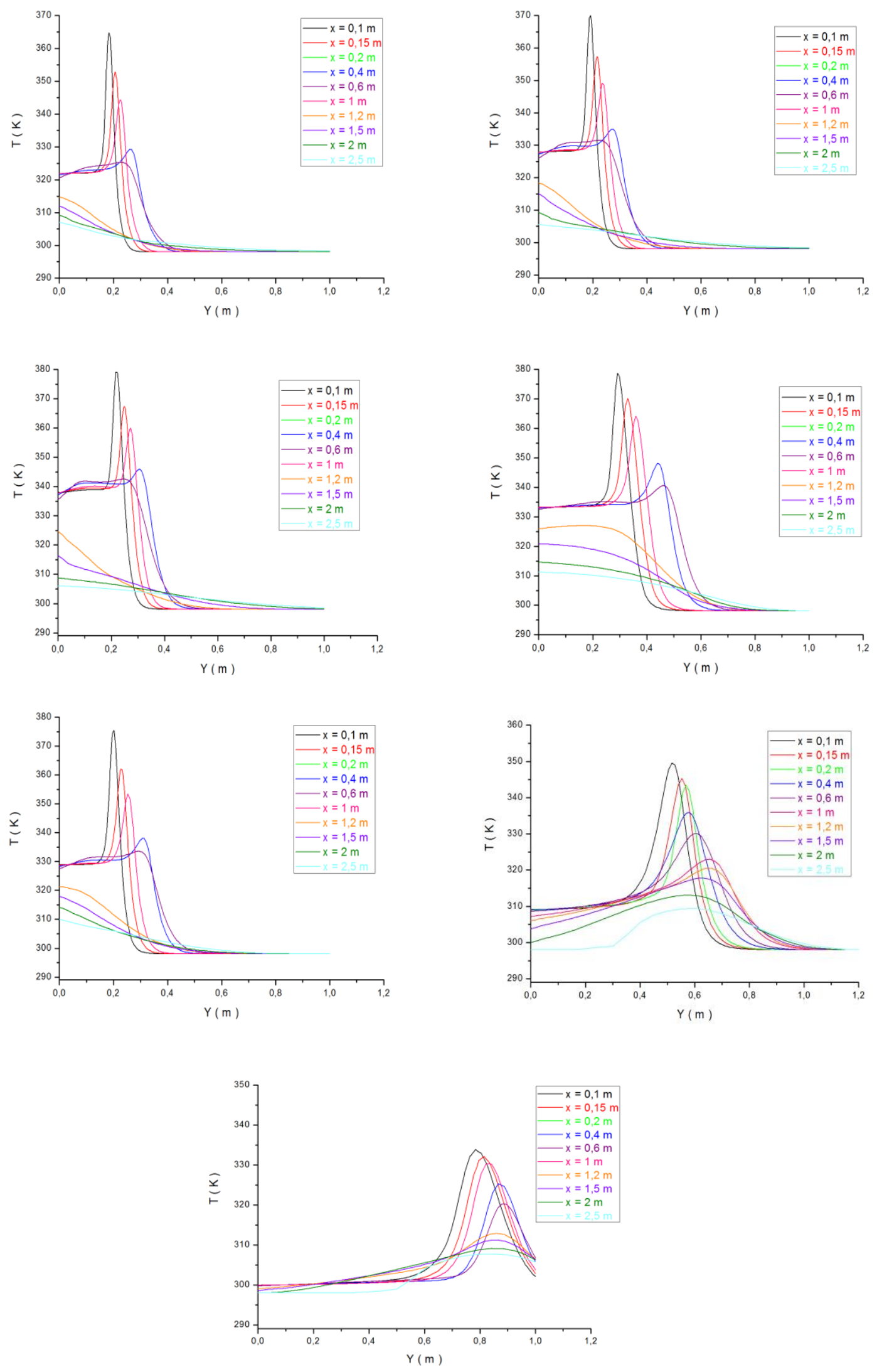

Figure 7. Influence of temperature for different values of $\alpha$ and at each position of $\mathrm{x}$ (straight stack) 


\section{Conclusions}

In this work, a numerical simulation of the turbulent flow from a straight chimney was performed. For this purpose, a 2D calculation code was used for an incompressible fluid. This code is based on a finite volume method to solve the complete Navier Stokes equations was used in this study. We first showed the comparison between our results obtained digitally with that of the literature in which a good agreement was illustrated.

Second the results found allow to determine the effect of the variation of several parameters on the flow such as wind speed direction and jet temperature. It has been shown that the greater the angle of the wind direction, the greater the dispersion of pollutants.

We also note that the temperature of the jet ejected from the chimney has a great effect on the dispersion. Indeed, the further away from the chimney the maximum temperature of the pollutant decreases.

In future studies, it is important to study the relationship between the diameter of the chimney and the dispersion of pollutants in the air. In addition, the study will be conducted under the variability of wind speed and direction.

\section{References}

1. N. Diaf, M. Bouchaour, L. Merad, B. Benyoucef .Paramètres influençant la dispersion des polluants gazeux. RevEnergRen : ICPWE 139-142 (2003).

2. B. Benkoussas et al . Etude de la dispersion atmosphérique des effluents émis par les cheminées de la cimenterie de Meftah. J Sci Res $\mathrm{N}^{\circ} 0$. 1:39-43 (2010).

3. M.E. Frankenberg \& T.T. Smith . Improvement of Ambient Sulfur Dioxide Concentrations by Conversion from Low to High Stacks. J Air Pollut Control Assoc . 25:595-601(1975).

4. D.J. Wilson . Turbulent dispersion in atmospheric shear flow and its wind tunnel simulation, Von Karman Institute for fluid dynamics, Technical note 76. (1971).

5. J.H. Vincent . Model experiments on the nature of air pollution transport near buildings. Atmos Environ . 11:765-774 (1977).

6. M. Menaouer . Modélisation numérique de l'évolution de la dispersion des polluants émis par une cheminée. Université des Sciences et de la Technologie Mohammed BOUDIAF-Oran (2013).

7. A.C. Flowe, A. Kumar . Analysis of velocity fields and dispersive cavity parameters as a function of building width to building height ratio using a 3-D computer model for squat buildings. J Wind Eng Ind Aerodyn . 86:87-122 (2000).

8. C. Liu \& G. Ahmadi . Transport and deposition of particles near a building model. Build Environ . 41:828-836 (2006).
9. H.A. Olvera, A.R. Choudhuri, W.W. Li . Effects of plume buoyancy and momentum on the near-wake flow structure and dispersion behind an idealized building. J Wind Eng Ind Aerodyn . 96:209-228 (2008).

10. S. DiSabatino et al . Simulations of pollutant dispersion within idealised urban-type geometries with CFD and integral models. Atmos Environ . 41:8316-8329 (2007).

11. Y. Tominaga \& T. Stathopoulos . Numerical simulation of dispersion around an isolated cubic building: Comparison of various types of $\mathrm{k}-\varepsilon$ models. Atmos Environ . 43:3200-3210 (2009).

12. B.E. Launder, D.B. Spaliding .The Numerical Computation of Turbulent Flows. Comput Methods Appl Mech Eng . 3:269-289 (1974).

13. S.V. Patankar .Numerical heat transfer and fluid flow. McGraw-Hill B Company, New York (1980).

14. S.N. Mahjoub, H. Mhiri, G. Le Palec, PH. Bournot. Experimental and numerical analysis of pollutant dispersion from a chimney. Atmos Environ. 39:1727-1738 (2005). 